\section{EPS International Conference on High Energy Physics, 27 June - 4 July}

Coincidental with the celebrations marking CERN's 25th Anniversary the 1979 EPS International Conference on High Energy Physics was held in Geneva. The attendance by more than eight hundred delegates and the significant amount of interesting new results testify to the vitality of the research in subnuclear physics.
At this conference everyone was looking forward to the first results from PETRA, the new $\mathrm{e}^{+} \mathrm{e}^{-}$storage ring at DESY, which started operating only a few months before. Data have been taken at $13 \mathrm{GeV}, 17 \mathrm{GeV}$ and, very recently, at $27.4 \mathrm{GeV}$. The total hadronic cross-section has been measured by three different groups and, within the large experimental uncertainties, they consistently find no evidence for any "step" which could indicate the appearence of a threshold for the production of a new quark. The hadronic jets that are assumed to arise from the fragmentation of the quark pairs that are produced, have been studied in detail. The measured properties of well explained by assuming that five quarks (u, d, s, c and b) are involved, and the authors claim that the inclusion of a sixth quark ( $t$ ) of electric charge $2 / 3$ and large mass, would produce a much poorer fit. Contrary to dence has been found for the production of $\mathrm{t} \overline{\mathrm{t}}$ pairs up to energies of 27.4 $\mathrm{GeV}$. The existence of this sixth quark is required in the standard WeinbergSalam model of the weak and electromagnetic interactions, in order to tary fermions, together with the bquark and the $\tau$-lepton, but there are no firm predictions on its mass. A slight excess of events with large sphericity has been found, which could be interpreted as being due to gluon radiation, while a few events with a three-jet configuration have been reported, in qualitative agreement with the expectations from quantum chromodynamics.

Beautiful results have been obtained from the study of the hadronic jets on the $\mathrm{Y}(9.4)$ resonance. The pattern of the hadronic energy flow looks very different from that off resonance and the distributions in several variables (thrust, sphericity, triplicity, etc.) are inconsistent with a pure phase-space effect. Instead, all these distributions are well fitted by assuming that the $\mathrm{Y}$ particle decays into three gluons, giving rise to three hadronic jets. These results are quite impressive since the decay of the $\mathrm{Y}$ into three gluons is a rather unique prediction of quantum chromodynamics. these jets (thrust, sphericity, etc.) are the hopes of many physicists, no evicomplete a third generation of elemen-
With regard to the charmonium family, the previously reported $\eta_{c}$ (2820) bound state has not been seen in a more accurate experiment, recently performed at SPEAR (SLAC).

The recent data on the neutral current weak interactions are all in nice agreement with the "standard", or "minimal", Weinberg-Salam model for the unification of the weak and electromagnetic interactions (hereafter denoted by $\mathrm{W}-\mathrm{S}$ ). The high statistics and good precision which have been achieved in the experiments with the CERN neutrino beams, have given a significant improvement in the determination of the only parameter in W-S (the Weinberg angle). New data have been reported from a difficult experiment performed at SLAC on the parity violation in the deep inelastic scattering of polarized electrons from deuterium, which are perfectly consistent with the W-S predictions. A recent mic transitions which has been perley, with larger experimental uncertainties) seems to agree with the expectations from W-S, contrary to some previous experiments. Altogether, the large variety of data which are now available allows a model-independent analysis to be made of the weak neutral current. The results of such an analysis are well described by W-S and tend to exclude many proposed alternatives.

As for the neutrino charged current deep inelastic interactions, the high statistics experiments (essentially those performed at CERN) have been analyzed in terms of the quark and gluon distributions inside the nucleons. Quantitative comparisons of the scaling violation observed in these structure functions, with the predictions of quantum chromodynamics have been attempted but the situation does not look conclusive yet. However, interesting information has been extracted from the data on the momentum distribution of the gluons inside the nucleon.

A striking discovery has been reported from a CERN experiment, with a $\pi^{-}$beam, where the $\psi / J$ particle is measurement of parity violation in atoformed at Novosibirsk (and at Berke-

\section{CLIMATIC VARIATIONS : FACTS AND CAUSES}

\section{ERICE, Sicily, 9-21 March 1980}

The first International School of Climatology (director, Prof. A. Longhetto) will be held at the Ettore Majorana Centre for Scientific Culture, Erice - Trapani, Sicily from 9 to 21 March 1980. It will deal with :

Climatic Changes and Variations: Facts, Causes and Geophysical Background. The main purpose of this course is to present a global overview of paleoclimatology, lectures being essentially oriented towards the physical basis of climatic changes and climatic variations.

This interdisciplinary course will provide an up-to-date survey of the most recent reconstructions of past climates and results of theoretical models simulating climatic changes and variations. Some lectures will also be devoted to man's impact on climate and a panel will discuss probabilities of climatic evolution in the next century.

The course is designed for people having a background in physical, mathematical, geophysical and/or meteorological aspects of phenomena occurring in the climatic system. The program has been made to provide information to researchers already working in this field as well as to stimulate and motivate all geophysicists in developments related to climatic variations. Lectures will be delivered by $\mathbf{2 5}$ specialists who will review the following subjects: Mathematical and Physical Basis of Climate, Reconstruction of Past Climates, Causes of Climatic Variations, Modelling Techniques and Man's Impact on Climate.

Some fellowships available for travel and/or living expenses will be awarded on a competitive basis. The number of participants will be limited. For further information and applications, contact the director of the course :

Prof. A. BERGER

Institute of Astronomy and Geophysics

Catholic University of Louvain, Chemin du Cyclotron 2

B-1348 Louvain-la-Neuve. 
copiously produced. A narrow peak has been found in the invariant-mass distribution of the $(\psi \mathrm{K} \pi)$ system, at $5.3 \mathrm{GeV}$. It could be interpreted as a meson carrying the newly discovered b-quark (plus a light quark). This meson (usually denoted by $B$ ) has thus a new quantum number, associated to the b-quark (otherwise called beauty). Although the branching ratio for the weak decay of the B-meson into $\psi \mathrm{K} \pi$ is expected to be of the order of $1 \%$, this final state provides a good signature. If the peak is confirmed, it can be considered as the first evidence for a particle carrying the beauty quantum number.

From the study of the massive muon pair production with $\pi$ beams, the momentum distribution of the quarks inside the $\pi$ has been determined for the first time, in three experiments. The results are in qualitative agreement with the theoretical expectations.

In the theoretical sessions, several imaginative speculations were discussed at the Conference in connexion with the intriguing question of what is going to happen in the vast region of energies between the weak boson masses, of the order of $10^{2} \mathrm{GeV}$, and the Planck mass of $10^{19} \mathrm{GeV}$ where the gravitational interaction becomes relevant. This, however, is looking far to the future and there is already plenty to think about.

\section{C.A. Savoy}

Geneva University

\section{Electromagnetic Interactions of Hadrons}

edited by A. Donnachie and G. Shaw

University of Manchester, England

\section{in two volumes}

Examining the definitive evidence photoproduction experiments have provided for quark model ideas, and the similarities between high-energy electromagnetic interactions and hadron-induced reactions, these volumes focus on photoproduction and electroproduction in the resonance region and at medium energies, multiparticle production and inclusive reactions, deep inelastic scattering, and electron-positron annihilation. Volumes in Nuclear Physics Monographs.

Volume 1: 458 pp., illus., $1978, \$ 59.40 / £ 31.19$ outside US

Volume 2: 590 pp., illus., $1978, \$ 69.00 / £ 36.23$ outside US

\section{New Frontiers in High-Energy Physics} edited by Arnold PerImutter and Linda F. Scott University of Miami, Florida

This volume surveys recent advances in high-energy physics stressing results in the study of strong interactions, and examining several areas that will prove especially significant in the coming years. Studies in the Natural Sciences, Volume 14. 680 pp., 1978, $\$ 71.40 / £ 37.49$ outside US

\section{Plenifinim}

227 West 17th Street, New York, N.Y. 10011 Black Arrow House, 2 Chandos Road London NW10 6NR, England

\section{Quantum Electronics Division}

The 1979 General Business Meeting for all members of the Quantum Electronics Division will be held at Poggio Imperiale, Florence, on 3 September at 21.00 , to coincide with the Divisional Meeting on Lasers in Photomedicine and Photobiology, organised by Professor R. Pratesi, Laboratorio di Elettronica Quantistica, Via Panciatichi 56/

\section{EPS 5th GENERAL CONFERENCE}

\section{7-11 September, 1981}

The EPS 5th General Conference will be held in Istanbul in collaboration with the Turkish Physical Society. Chairman of the International Programme Committee is E. Matthias of Berlin (West) and of the Local Organizing Committee, E. Inönü of the Bogaziçi University, Istanbul.
30, I- 50127 Florence, tel. (55) 416128

The Quantum Electronics Division will be acting as an Advisory Programme Committee for the International Quantum Electronics Conference in Boston 1980. The Chairman (Professor S.D. Smith, Department of Physics, Heriot-Watt University, Riccarton, Currie, Edinburgh EH14 4AS, UK, telephone (0) 31-449 5542) would like to hear about any important work, worthy of inclusion.

The Quantum Electronics Division will be organising the First European Conference on Optical Systems and Applications in Utrecht from 23 to 25 September 1980. The chairman is Dr B. Bölger, Philips Research Laboratories, NL - 4500 Eindhoven, telephone (040) 742179 .
EPS Dlvisions, Sections and Group

Astronomy and Astrophysics Division Solar Section

Atomic Physics Division

Atomic Spectroscopy Section

Chemical Physics

Electronic and Atomic Collisions

Molecular Physics

Computational Physics Group

Condensed Matter Division

Low Temperature Section

Macromolecular Physics

Magnetism

Metals

Semiconductors and Insulators

Surface and Interface

High Energy \& Particle Physics Division

Nuclear Physics Division

Plasma Physics Division

Quantum Electronics Division
Europhysics News is the official journal of the European Physical Society that comprises $28 \mathrm{Na}$ tional Societies. Academies and Groups, over tional Societies, Academies and Groups, over
3000 Individual Ordinary Members and 30 Asso3000 Individual Ordinary Members and 30 Asso-
ciate Members. Governing bodies of EPS are the General Meeting, Council and an elected Executive Committee responsible for detailed policy EPS promotes the collaboration of physicists EPS promotes the collaboration of physicists throughout Europe and encourages all aspects of international exchange in physics. EPS awards scholarships for research and studies in different countries. EPS publishes, in addition to Europhys ics News, Europhysics Conference Abstracts, Europhysics Education News and the Proceedings of its General Conferences. Individual Ordinary Members receive Europhysics News (subscription (or non for non-Memb tes on many publications and pay reduced fees at conferences. Application for membership is made through the permanent Secretariat which is Jocated in Geneva. Annual subscription for members of a National Society is $28 \mathrm{Sw} . \mathrm{Fr}$.
Editor: E.N. Shaw

Meetings Compilation: W.S. Newman

Editorial Board

K. Appert, G.J. Béné, G.R. Macleod,

A. Maeder, J. Muller, D. Pohl

Editorial and Advertising Office at the EPS Secretariat.

Address: EUROPEAN PHYSICAL SOCIETY P.O. BoX 69, CH-1213 Petit-Lancy 2 Switzerland

Telephone: Geneva (22) 931130

Telex : 23455 alarm ch

Cables: europhys genève

Printed by : Ed. Cherix et Filanosa SA CH-1260 Nyon, Switzerland 